\title{
An Extensive Estimation and Analysis of Image Denoising
}

\author{
Prerna Rajput \\ M-Tech Research Scholar, \\ Department of Computer Science, \\ TIT, Bhopal
}

\author{
Shiv Kumar Singh Tomar \\ Asst. Prof. and Guide, \\ Department of Computer Science, \\ TIT, Bhopal
}

\author{
Bhupesh Gour, Ph.D. \\ Professor and HOD, \\ Department of Computer Science, \\ TIT, Bhopal
}

\begin{abstract}
Image denoising is the technique of removal of the noise from the image contaminated by additive Gaussian noise without loss of features of image. It is a fundamental process in pattern recognition and image processing. When an Image is captured many factors such as lighting spectra, source, intensity and camera Characteristics affect the image. The main factor that reduces the quality of the image is Noise. It hides the important information of images and changes value of image pixels at key locations causing blurring and various other deformities. Noises must be removed from the images without loss of any information with it. Noise removal is the preprocessing stage of image processing. There are many types of noises which may corrupt the images. These noises are appear on images in many ways: at the time of acquisition due to noisy sensors, due to defective scanner or due to faulty digital camera device, as a result of transmission channel errors, due to corrupted storage media. There are numerous researches have been done on wavelet based denoising for estimation of parameters such as variance of the multi scale Linear minimum mean square error. In this review paper we have presented an extensive analysis and literature review on image denoising.
\end{abstract}

\section{Keywords}

Optimization; Image denoising; Image restoration \& TV model

\section{INTRODUCTION}

Image denoising is the problem of restoring a clean image from a noisy image [1]. In most cases, it is assumed that the noisy or corrupted image is the summation of original image and a noise component, as shown in Fig. 1. Hence denoising is a procedure which can remove the existing noise in an image and minimizes the loss of features in a clean image. It includes prior knowledge: Without prior knowledge, image denoising would not be possible. In case of denoising the characteristic of the system as well as the type of noise is known beforehand. The image $\mathrm{s}(\mathrm{x}, \mathrm{y})$ is blurred by the linear operations causing the noise $\mathrm{n}(\mathrm{x}, \mathrm{y})$ to add or multiply among the image. The image denoising model:

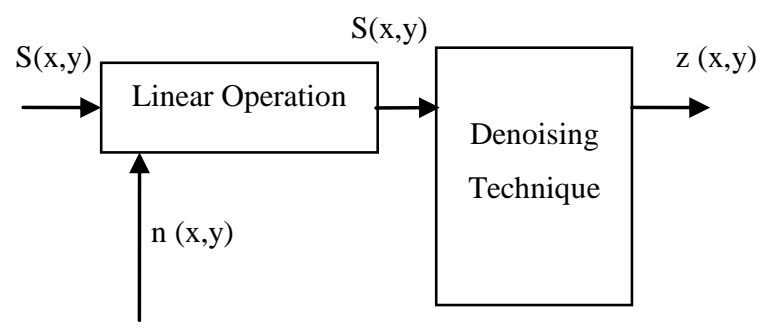

Fig. 1 Model of Image Denoising
The noisy image then undergoes a denoising procedure and produces the denoised image $\mathrm{z}(\mathrm{x}, \mathrm{y})$. How close the image $\mathrm{z}(\mathrm{x}, \mathrm{y})$ is to the original image depends on the noise levels and the denoising algorithm use [9].

\subsection{Significant of Denoising}

Image Denoising is the part of preprocessing in Pattern Recognition system. The digital images sensed by sensors are generally corrupted by Gaussian noise during the process of acquisition, retrieval and transmission from storage media. During the image acquisition process and interference noise may be generated due to improper settings of sensors used in image processing. A noisy image is not satisfying to view. Therefore, it is very important to get the improved image from the corrupted image without loss of features of the image. In Pattern recognition it need a denoised image to work effectively. Random and uncorrelated noise samples are not compressible. Thus the motivation behind the denoising algorithm is to remove Gaussian noise. This entire factor can give importance of denoising in image processing.

\subsection{Types of Noises in Images}

Noise in image is caused by fluctuations in the brightness or color information at the pixels. Noise is a process that distorts the acquired image and is not a part of the original image. Noises in images can take place in many ways. Throughout image acquisition the optical signals get converted into electrical which then gets converted to digital signal [10]. At each process of conversion noise gets added to the image. The image may also have noisy during transmission of the image in the form of digital signals. Types of noises are given:

1. Gaussian noise

2. Salt and Pepper noise

3. Shot noise (Poisson noise)

4. Speckle noise

\subsection{Gaussian Noise}

Has normal Gaussian distribution. It is consistently distributed over the image signal. Gaussian distribution is given by

$$
F(g)=\frac{1}{2 \Pi \sigma^{2}} e^{-(g-m) 2 / 2 \sigma^{2}}
$$

g- Grey level

m- Mean of function

Sigma- standard deviation of noise 


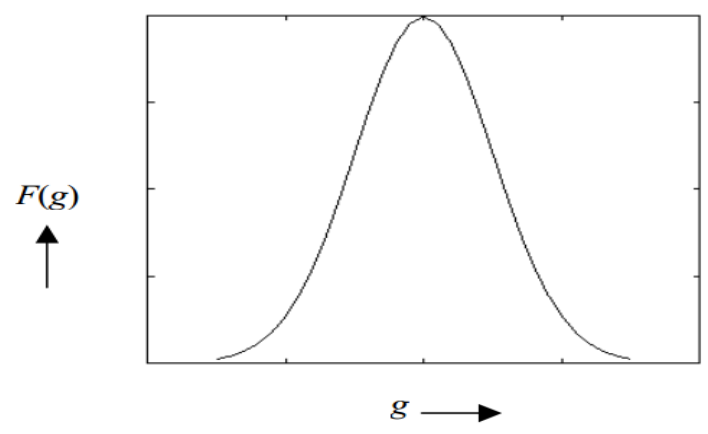

Fig.2 Bell Shaped Curve for Gaussian distribution

\subsection{Salt and Pepper Noise}

It is an impulse type noise comprising of intensity spikes. It has two possible values whether $a$ or $b$ the probability of each of the values is less than 0.1 . The pixels may corrupted with salt and pepper noise alternate between the minimum and maximum value it causes randomly occurring black and white pixels. The major causes of this type of noise are malfunctioning of pixel elements in camera and malfunctioning of analog to digital conversion in camera.

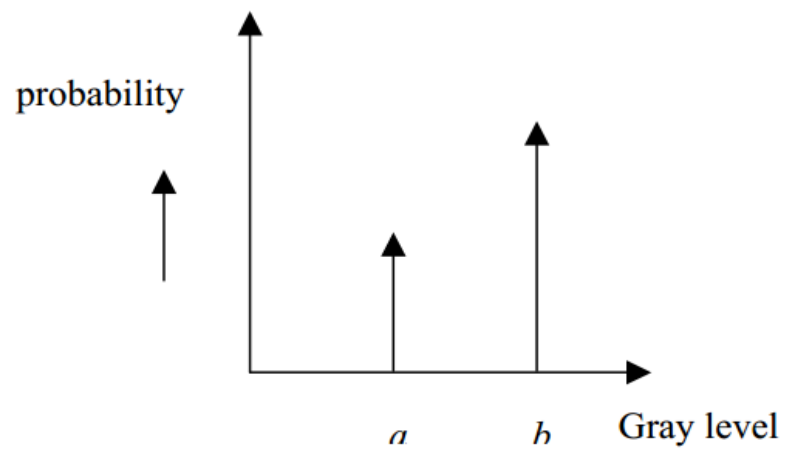

Fig. 3 Salt and Pepper Noise

\subsection{Speckle Noise}

It is a multiplicative noise occurring mostly in medical images in all coherent imaging systems like laser, acoustics ultrasound etc. It follows gamma distribution.

$$
F(g)=\frac{g^{\alpha-1}}{(\propto-1) ! \alpha^{\alpha}} e^{-\frac{g}{a}}
$$

Where $\mathrm{a}$ is standard deviation and alpha and $\mathrm{g}$ are the grey levels

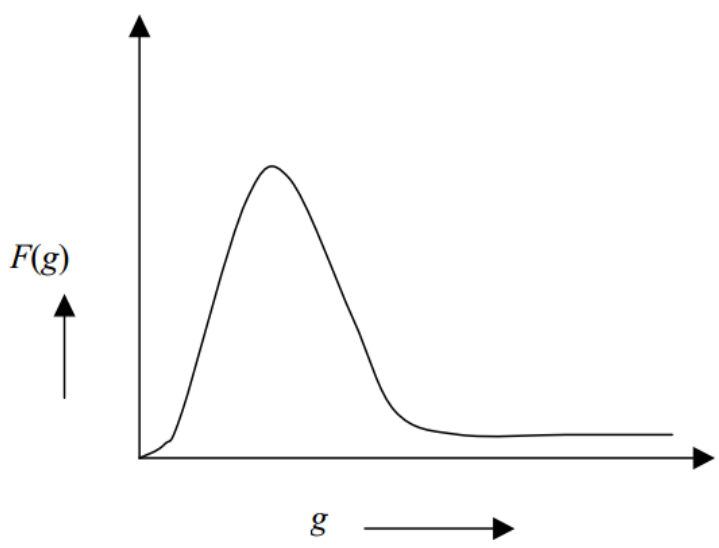

Fig. 4 Gamma Distribution

\subsection{Brownian Noise}

Brownian noise comes under the category of fractal or $1 / \mathrm{f}$ noises. The arithmetical model for $1 / \mathrm{f}$ noise is fractional Brownian motion. Fractal Brownian motion is a nonstationary stochastic procedure that follows a normal distribution. Brownian noise is a unique case of $1 / \mathrm{f}$ noise

Fig. 5

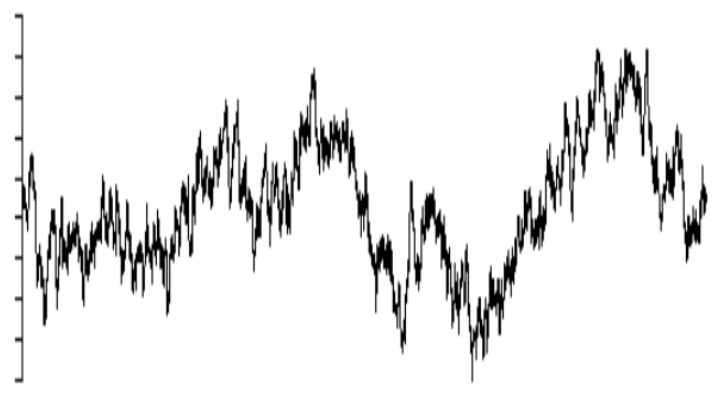

Fig. 5 Brownian Noise Distribution

\section{SYSTEM MODULE}

It is a simple sliding window spatial filter. It can replace the center value of the window with average of all the pixels in the window. The convolution mask provides weighted sum of values of pixels [9] and its neighbors. The convolution mask is square and works on the shift multiply sum principle as depicted:

This convulsion mean filtering is effective when the noise in impulsive. The mean filter acts like a low pass filter and does not allow the high noise frequencies to pass through.
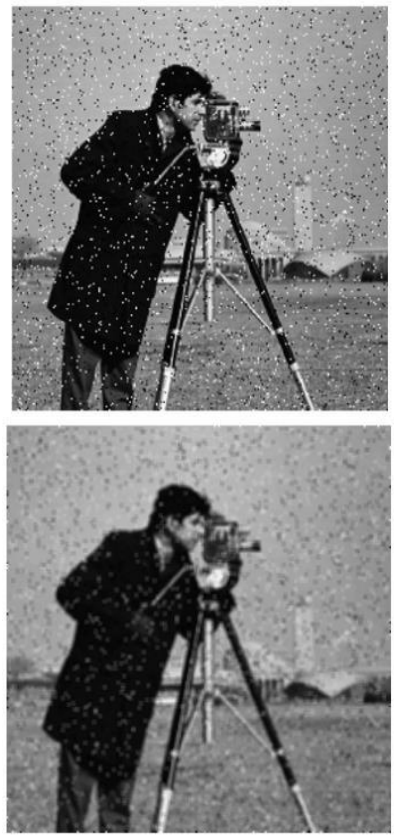

Fig. 6 Input Image with salt and Pepper Noise Output Image

\subsection{LMS Adaptive Filter}

The weighted sum is obtained by: The main difference between mean filter and an adaptive filter is that in an adaptive filter, weighted matrix changes after every iteration. It is helpful for images with variable noise and can be applied to unknown image type without the knowledge of the type of 
noise present in it. This is the combination of non-changing low pass filter and a changing [10] high pass filter. It works in the following manner:

Window of size $a * b$ is chosen above images, $u$ is the mean of the window, it is deduct from each element of the window and the resultant matrix is $\mathrm{Wr}$.

$$
\begin{aligned}
& W r=W-u \\
& z=\sum_{(i, j)} h(i, j) W^{r}
\end{aligned}
$$

$\mathrm{H}(\mathrm{i}, \mathrm{j})$ element of the weighted matrix. The addition of $\mathrm{z}$ and mean of window change along with the center value of window. The pixel value now is:

$$
Z=Z+\text { ? }
$$

The next step the window has been changed one pixel in row major order and the weight matrix is modified as follows:

$$
e=W^{r}-z^{\sim}
$$

$\mathrm{E}$ is the deviation. Largest eigen value of the original window is calculated.

$$
0<\eta<1 / \lambda
$$

The new weighted matrix is:

$$
h_{k+1}=h k+\eta * e * W^{r}
$$

This is used in the next iteration. The process continues up till the entire image is covered.
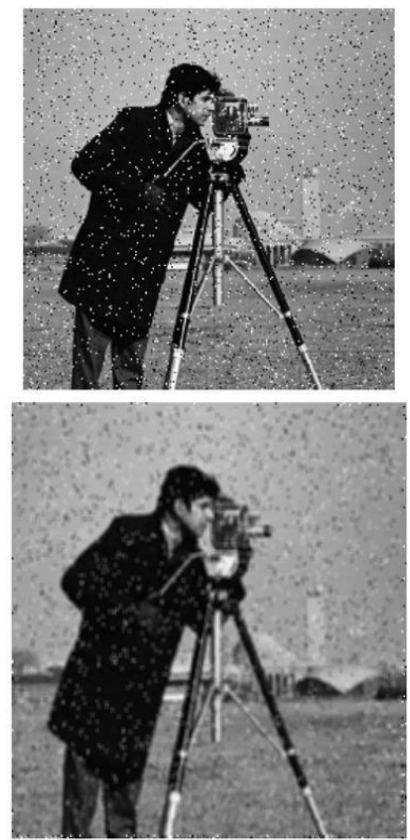

Fig. 7 Image with Salt and Pepper Noise Output Image

\subsection{Median Filter}

It is a nonlinear filter. Has the same sliding window concept but the center pixel value is exchanged with the median of the neighboring pixels. Every pixel is sorted numerically and then the center value is exchanged with the median of the window [13]. Median filter is very resistant towards pixel value with unusual values not matching the pixel values in the image.
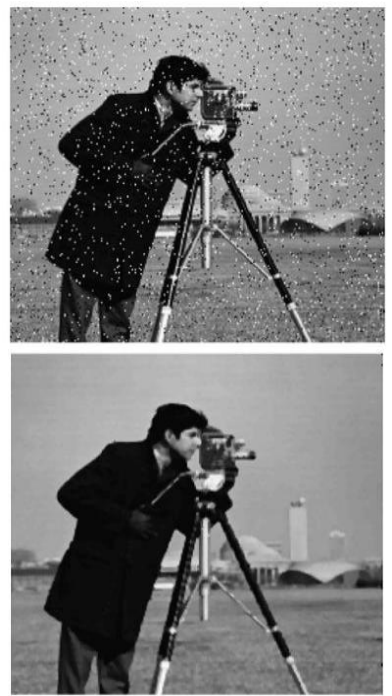

Fig. 8 Input Images and Output Image

\section{LITERATURE REVIEW}

Leifu Gao and Chao Li [1] investigated three important denoising models: the harmonical model, the TV (i.e. total variation) model and the general TV model, authors presented an adaptive one which is named adaptive TV image denoising model'. This model can pretreat them with Gaussian filter, as a result as to overcome the staircase effect in the TV model. Then by utilize the gradient information of every pixel point of the image, they can adaptively select the most appropriate denoising scheme. The results of experiments show that this method can preserve significant image details while removing the noise and compared with other denoising methods, particularly at high noise levels, this technique achieves at least about 1.0dB gain for PSNR measurement.

Yao Zhao, Jianguo Liu and Bingchen Zhang [2] introduced a total variation (TV) regularization model for SAR image despeckling. The TV regularization based image restoration model has a good performance in preserving image sharpness and edges while removing noises and it is therefore effective for edge preserve SAR image despeckling. Results have been carried out using optical images contaminated with artificial speckles first and then SAR images. Result shows that the proposed method can effectively suppress SAR image speckles without compromise the edge sharpness of image features according to both subjective visual examination and objective evaluation indices of image quality.

Boyat A. and Joshi B.K. [3] presented acquisition of an image, from the source; noise becomes integral part of it, which is very difficult to remove. A new image denoising algorithm based on combined effect of wavelet transform and median filtering. The algorithm removes most of the noisy part from the image and maintains the quality. The point of wavelet decomposition is restricted to three. The renowned index PSNR and RMSE demonstrate marked improvement of image denoising over other methods.

Huijie Guo and Weihai Fang; [4], have done research in the field of image enhancement. An image badly lack of details, the solution of image enhancement is to reconstruct the unknown original details in terms of the small amount of known information. To entirely or approximately reconstruct an unknown signal by a small number of its known elements is a matrix completion problem in the sparse theory. Authors 
propose an image enhancement algorithm based on matrix completion that implements the effective complements of local details to the local blurred image by solving the nuclear norm minimization problem with the method of singular value shrinkage iteration, and gets image enhancement with fine subjective qualities for human vision.

Veeramani, T., Rajagopalan, A.N. and Seetharaman, G., [5] worked on image defogging technique that can restore contrast loss and yield a rough estimate of the depth map of a scene. Imaging devices has attracted considerable attention to motion blur but this has not been addressed in the context of images captured under foggy conditions. Authors restore foggy motion-blurred images using depth cues derived from fog itself. Initially, they address restoration of images blurred primarily due to in-plane translational camera motion. They demonstrate that foggy road scene images can be segmented into road, right, left and sky planes, and that each of these planes can be deblurred individually.

Gibson, K.B. and Nguyen, T.Q. [6] They presented a fast single image defogging method that uses a novel approach to refining the estimate of amount of fog in an image with the Locally Adaptive Wiener Filter. They provide a solution for estimating noise parameters for the filter when the observation and noise are correlated by decorrelating with a naively estimated defogged image. They demonstrate our method is 50 to 100 times faster than existing fast single image defogging methods and the proposed method subjectively performs as well as the Spectral Matting smoothed Dark Channel Prior method.

Ota, A., Yoshida, T. and Ikehara, M., [7] investigated a novel de-blocking algorithm, using block wiener filtering, shift and weighted average operations. The dissipation of block edge occurs by a combination of the shift and average operations. Visually annoying artifacts are noticeable around the edge region in highly compressed images. In this method, the wiener attenuation coefficients are given based on the estimated error in a block unit. The DCT encoded images gets a large margin of error from one block to another.

Table 1: Summary of Literature Review

\begin{tabular}{|c|c|c|c|c|}
\hline Year & Author & Title & Approach & Result \\
\hline 2013 & $\begin{array}{l}\text { Leifu Gao } \\
\text { Chao Li }\end{array}$ & $\begin{array}{l}\text { An Adaptive TV Model for Image } \\
\text { Denoising }\end{array}$ & $\begin{array}{l}\text { Design the building of } \\
\text { the adaptive TV model } \\
\text { for image denoising. }\end{array}$ & $\begin{array}{l}\text { Authors use images of } \\
\text { Angela and Place which } \\
\text { are polluted by noise as } \\
\text { input objects. Their sizes } \\
\text { are } 256 \times 256 \text {, and gray } \\
\text { levels } 256 \text {. }\end{array}$ \\
\hline 2013 & $\begin{array}{l}\text { Yao Zhao, Jianguo } \\
\text { Liu Bingchen Zhang } \\
\text { Wen Hong Yirong } \\
\text { Wu }\end{array}$ & $\begin{array}{l}\text { An Adaptive total variation } \\
\text { regularization method for sar } \\
\text { image despeckling }\end{array}$ & $\begin{array}{lr}\text { Design of } & \text { TV } \\
\text { Regularization } & \text { based } \\
\text { SAR } & \text { image } \\
\text { despeckling. } & \end{array}$ & $\begin{array}{l}\text { Images are contaminated } \\
\text { by adding white Gaussian } \\
\text { noise with variance of } 18 .\end{array}$ \\
\hline 2013 & $\begin{array}{l}\text { Ajay Boyat } \\
\text { Brijendra Kumar } \\
\text { Joshi }\end{array}$ & $\begin{array}{l}\text { Image Denoising using Wavelet } \\
\text { Transform and } \\
\text { Median Filtering }\end{array}$ & $\begin{array}{l}\text { Design of a Wavelet } \\
\text { Transform for image } \\
\text { Denoising }\end{array}$ & $\begin{array}{l}\text { Authors have mainly } \\
\text { focused on iterative noise } \\
\text { density and median filter } \\
\text { for determining the PSNR } \\
\text { and RMSE using Gaussian } \\
\text { noise model. }\end{array}$ \\
\hline 2013 & $\begin{array}{l}\text { Huijie Guo, Weihai } \\
\text { Fang, Xin Wen, Feng } \\
\text { Nian }\end{array}$ & $\begin{array}{l}\text { Image enhancement based on } \\
\text { matrix completion }\end{array}$ & $\begin{array}{l}\text { Design of a matrix } \\
\text { Completion } \\
\text { reconstruction }\end{array}$ & $\begin{array}{l}\text { Implement the matrix } \\
\text { completion reconstruction } \\
\text { by virtue of the singular } \\
\text { value shrinkage iteration } \\
\text { algorithm; }\end{array}$ \\
\hline 2013 & $\begin{array}{l}\text { Thangamani } \\
\text { Veeramani } \\
\text { Ambasamudram N. } \\
\text { Rajagopalan Guna } \\
\text { Seetharaman }\end{array}$ & $\begin{array}{l}\text { Restoration of foggy and motion- } \\
\text { blurred road scenes }\end{array}$ & $\begin{array}{l}\text { Design of a Motion } \\
\text { Deblurring in fog }\end{array}$ & $\begin{array}{l}\text { They obtained the blurred } \\
\text { radiance and depth map of } \\
\text { the scene using the dark } \\
\text { channel prior }\end{array}$ \\
\hline 2013 & $\begin{array}{l}\text { Kristofor B. Gibson } \\
\text { and Truong Q. } \\
\text { Nguyen }\end{array}$ & $\begin{array}{l}\text { Fast single image fog removal } \\
\text { using the adaptive wiener filter }\end{array}$ & $\begin{array}{l}\text { Designed for near real- } \\
\text { time speeds for videos } \\
\text { with resolution near } \\
720-480 \text { resolution. }\end{array}$ & $\begin{array}{l}\text { They obtained the } 21 \\
\text { images processed with } \\
\text { resolutions ranging from } \\
465 \_384 \text { to } 3072 \_2304 \text {. }\end{array}$ \\
\hline 2013 & $\begin{array}{l}\text { Azusa Ota, Taichi } \\
\text { Yoshida, and } \\
\text { Masaaki Ikehara }\end{array}$ & $\begin{array}{l}\text { Blocking Artifacts Reduction of } \\
\text { DCT Compressed } \\
\text { Image Based on Block Wiener } \\
\text { Filtering }\end{array}$ & $\begin{array}{l}\text { Designed for DE- } \\
\text { blocking algorithm via } \\
\text { block wiener filtering }\end{array}$ & $\begin{array}{l}\text { They demonstrate the } \\
\text { performance of the } \\
\text { proposed algorithm. The } \\
\text { test images are Barbara, } \\
\text { Pepper and Lena, these } \\
\text { standard } 512 \times 512 \text { [pixel] } \\
\text { 8- bit grayscale images. }\end{array}$ \\
\hline
\end{tabular}


Therefore texture information losses are prevented while smoothening effect is applied to the region around the edge.

\section{PROBLEM FORMULATION \\ 4.1 Noise Model}

An Image processing block enhances the image and compensates for some of the deficiencies of the other camera blocks. The memory is present to store the image, while a display may be used to preview it. There are some blocks exist for the purpose of user control. Noise may be added to the image in the lens and ADC as well as in the image processing block itself. The sensor is made of millions of tiny light-sensitive components [1]. They differ in their physical and electrical, which add a signal-independent noise, to the acquired image. Another component of shot noise is the photon shot noise. That occurs due to the number of photons detected varies across different parts of the sensor. Amplification of the sensor signals adds amplification noise, which is zero mean Gaussian noise. There are other types of noise exist. Correlated noise with a Guassian distribution is an example. Noise may also have different distributions such as Laplacian, Poisson, or non-additive Salt-and-Pepper noise. It is caused by bit errors in image transmission and retrieval as well as in analog-to-digital converters.

The problem of denoising can be mathematically represented as given,

$$
Y=X+\eta
$$

Let $\mathrm{X}$ be a original image with size $\mathrm{N} \times \mathrm{N}, \mathrm{Y}$ be a observed noisy image and be zero-mean Gaussian noise with variance 2 , where $2 \mathrm{~N}(0,2)$ The objective is to estimate true image $X$ from given noisy image $\mathrm{Y}$. A best estimate can be written as the conditional mean.

The problem lies in determining the probability density function $(\mathrm{x} \mid \mathrm{y})$; the goal is to estimate the true image $\mathrm{X}$ from the noisy image $\mathrm{Y}$ without loss of features of the original image.

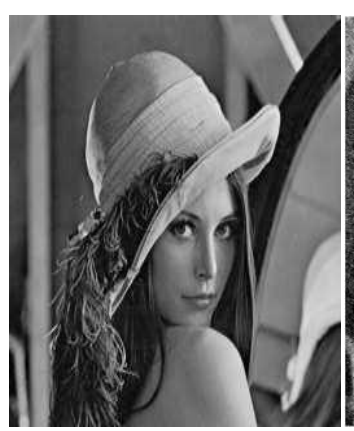

(a)

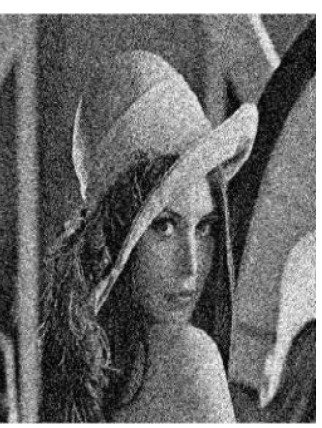

(b)
Fig. 9: (a) Original Image. (b) Noisy Image

\section{RESEARCH MOTIVATIONS}

Multiplicative noise or speckle noise occurred in various imaging systems due to random variation of pixel values. Though a number of restoration techniques were used in literature like Wiener filtering and Lee filtering to denoise such kind of noisy images, however these methods are not giving promising results in terms of PSNR, MSE and SNR. Various thresholding techniques can be used like hard thresholding and entropy based thresholding in curvlet domain to denoise these images.

\section{CONCLUSIONS AND FUTURE SCOPE}

Image denoising using the existing denoising algorithms using various different approaches have been reviewed in a literature review. New proposed filtration and estimator method would be implemented for image denoising. Different estimation expression and different experimental results has been studied. This scheme gives fairly satisfying results in both PSNR. There are several well known algorithms for denoising the images and their performance was comparatively assessed in the research directions which have scope of further investigation.

\section{REFERENCES}

[1] Leifu Gao; Chao Li, "An adaptive TV model for image denoising," Natural Computation (ICNC), 2013 Ninth International Conference on , vol., no., pp.766,770, 23 25 July 2013.

[2] Yao Zhao; Jianguo Liu; Bingchen Zhang; Wen Hong; Yirong $\mathrm{Wu}$, "An adaptive total variation regularization method for SAR image despeckling," Geoscience and Remote Sensing Symposium (IGARSS), 2013 IEEE International , vol., no., pp.3084,3087, 21-26 July 2013.

[3] Boyat, A.; Joshi, B.K., "Image denoising using wavelet transform and median filtering," Engineering (NUiCONE), 2013 Nirma University International Conference on , vol., no., pp.1,6, 28-30 Nov. 2013.

[4] Huijie Guo; Weihai Fang; Xin Wen; Feng Nian, "Image enhancement based on matrix completion," Cross Strait Quad-Regional Radio Science and Wireless Technology Conference (CSQRWC), 2013 , vol., no., pp.357,360, 21-25 July 2013.

[5] Veeramani, T.; Rajagopalan, A.N.; Seetharaman, G., "Restoration of foggy and motion-blurred road scenes," Image Processing (ICIP), 2013 20th IEEE International Conference on , vol., no., pp.928,932, 15-18 Sept. 2013.

[6] Gibson, K.B.; Nguyen, T.Q., "Fast single image fog removal using the adaptive Wiener filter," Image Processing (ICIP), 2013 20th IEEE International Conference on , vol., no., pp.714,718, 15-18 Sept. 2013.

[7] Ota, A.; Yoshida, T.; Ikehara, M., "Blocking artifacts reduction of DCT compressed image based on block wiener filtering," Advanced Technologies for Communications (ATC), 2013 International Conference on , vol., no., pp.85,89, 16-18 Oct. 2013.

[8] Coifman, Ronald R., and David L. Donoho. Translationinvariant de-noising. Springer New York, 1995.

[9] Chang, S. Grace, Bin Yu, and Martin Vetterli. "Adaptive wavelet thresholding for image denoising and compression." Image Processing, IEEE Transactions on 9.9 (2000): 1532-1546.

[10] Om, Hari, and Mantosh Biswas. "A generalized image denoising method using neighbouring wavelet coefficients." Signal, Image and Video Processing (2013): $1-10$

[11] Sendur, Levent, and IvanW. Selesnick. "Bivariate shrinkage with local variance estimation." Signal Processing Letters, IEEE 9.12 (2002): 438-441.

[12] Pizurica, Aleksandra, et al. "A joint inter-and intrascale statistical model for Bayesian wavelet based image 
denoising." Image Processing, IEEE Transactions on 11.5 (2002): 545-557.

[13] Hou, Zujun. "Adaptive singular value decomposition in wavelet domain for image denoising." Pattern Recognition 36.8 (2003): 1747-1763.

[14] Boubchir, Larbi, and Boualem Boashash. "Wavelet denoising based on the MAP estimation using the BKF prior with application to images and EEG signals." (2013): 1-1.

[15] Zhang, Guo-Duo, et al. "Image denoising based on support vector machine." Engineering and Technology (S-CET), 2012 Spring Congress on. IEEE, 2012.

[16] Chen, G. Y., Tien D. Bui, and Adam Krzyzak. "Image denoising using neighbouring wavelet coefficients." Integrated Computer-Aided Engineering 12.1 (2005): 99-107.

[17] Chen, G. Y., and T. D. Bui. "Multiwavelets denoising using neighboring coefficients." Signal Processing Letters, IEEE 10.7 (2003): 211-214.

[18] Ruikar, Sachin, and D. D. Doye. "Image denoising using wavelet transform."Mechanical and Electrical Technology (ICMET), 2010 2nd International Conference on (pp 509-515).IEEE, 2010.

[19] Jain AK. Fundamental of digital image processing.Prentice Hall;Upper Saddle River,NJ;2001.
[20] Liua, Jia, Caicheng Shi, and Meiguo Gao. "Image denoising based on BEMD and PDE." Computer Research and Development (ICCRD), 2011 3rd International Conference on. Vol. 3. IEEE, 2011.

[21] Om, Hari, and Mantosh Biswas. "MMSE based map estimation for image denoising." Optics Laser Technology 57 (2014): 252-264.

[22] Jiang, Jun, et al. "An improved adaptive wavelet denoising method based on neighboring coefficients." Intelligent Control and Automation (WCICA), 2010 8th World Congress on. IEEE, 2010.

[23] Donoho, David L. "Unconditional bases are optimal bases for data compression and for statistical estimation." Applied and computational harmonic analysis 1.1 (1993): 100-115.

[24] Mallat, Stephane G. "A theory for multiresolution signal decomposition: the wavelet representation." Pattern Analysis and Machine Intelligence, IEEE Transactions on 11.7 (1989): 674-693.

[25] Abramovich, Felix, Theofanis Sapatinas, and BernardW. Silverman. "Wavelet thresholding via a Bayesian approach." Journal of the Royal Statistical Society: Series B (Statistical Methodology) 60.4 (1998): 725-749 